\title{
FSH response-dose can be predicted in ovulation induction for normogonadotropic anovulatory infertility
}

\author{
Evert J P van Santbrink, Marinus J C Eijkemans ${ }^{1}$, Nicholas S Macklon and Bart C J M Fauser \\ Division of Reproductive Medicine, Department of Obstetrics and Gynecology and ${ }^{1}$ Center for Clinical Decision Sciences, Department of Public Health, \\ Erasmus Medical Center, The Netherlands
}

(Correspondence should be addressed to B C J M Fauser, Department of Obstetrics and Gynecology, Erasmus Medical Center, Dr Molewaterplein 40, 3015 GD, The Netherlands; Email: fauser@gyna.azr.nl)

\begin{abstract}
Objective: To evaluate the ability of a prediction model to identify the individual starting dose of FSH for ovulation induction using a step-down regimen.

Design: Retrospective analysis of clinical data in an academic fertility unit. Fifty-six normogonadotropic anovulatory infertile patients who failed to ovulate or conceive with clomiphene citrate were included. They were treated with exogenous gonadotropins with a flexible starting dose for ovulation induction using a step-down regimen. The clinically applied starting dose of exogenous gonadotropins was compared with the calculated response-dose using a previously published prediction model.

Results: Patients were arbitrarily divided into three groups according to the day of the first decrease in gonadotropin dose: (a) early step-down (day 3 or earlier); (b) standard step-down (day 4 or later); (c) no step-down. These groups had average starting doses of 28.5 IU (group a) and 13 IU (group b) above the calculated response-dose, and 43 IU (group c) under the calculated response-dose. A significant correlation between day of first step-down and the difference between clinically applied and calculated response-dose was observed ( $P<0.0001$, F-test for ANOVA).

Conclusions: The patient group with the best step-down profile for ovulation induction exhibited the closest match between the clinically applied and calculated starting dose of gonadotropins. Therefore, this study provides support for the concept that the individual effective FSH starting dose for gonadotropin induction of ovulation in anovulatory infertile patients can be predicted on the basis of initial screening characteristics, such as body mass index, clomiphene resistance or failure, free IGF-I and FSH. This may result in more effective patient treatment protocols, reduced complication rates and health-economic benefits.
\end{abstract}

European Journal of Endocrinology 147 223-226

\section{Introduction}

The principal risks associated with gonadotropin induction of ovulation are multiple pregnancy and ovarian hyperstimulation syndrome (OHSS) $(1,2)$. Both of these complications arise from multiple follicle growth. This may occur when the 'threshold' level of follicle-stimulating hormone (FSH) for ongoing follicular growth is exceeded for too long, extending the FSH 'window' for a sufficient period of time to allow for multi-follicular dominance $(3,4)$. In order to reduce complication rates, single, intermittent or multiple fixed dose as well as incremental or decremental dose regimens have been proposed (5-8). At present, the most frequently used administration schedule is the low-dose step-up regimen in which the dose of exogenous gonadotropins is gradually increased until ovarian response is observed $(7,9)$. However, this can be a time-consuming approach, especially in women with a high FSH threshold. An alternative dose regimen developed by our group is the step-down protocol (8, 10 ) in which a relatively high initial starting dose of FSH is followed by subsequent small decremental steps. This results in a serum FSH profile that mimics more closely physiological conditions in normo-ovulatory women $(11,12)$. The major disadvantage of this regimen, however, is that the initial fixed FSH dose may be too high for some women resulting in enhanced chances of complications and cycle cancellations (13). We recently described a prediction model using initial patient characteristics for the individual FSH starting dose in low-dose regimens for ovulation induction (14). The ability to assess the effective individual FSH response-dose would improve the course and outcome in both low-dose step-up and step-down protocols by reducing the duration of treatment and complication rates. It may also make the protocols more costeffective. The aim of the present study was to determine 
retrospectively whether this model would be able to predict when the used starting dose had been appropriate or too high or too low for an individual patient who had undergone a step-down protocol for induction of ovulation.

\section{Materials and methods}

\section{Subjects and study design}

Between 1993 and 199656 patients attending our fertility unit meeting the following criteria were included in the study: (i) oligomenorrhea (bleeding intervals between 35 days and 6 months) or amenorrhea (bleeding interval $>6$ months), (ii) serum FSH levels within normal limits (1-10 IU/l (11)), (iii) normal serum prolactin and thyroid-stimulating hormone levels, (iv) spontaneous menses or positive bleeding response to progestagen withdrawal, (v) infertility (seeking pregnancy $\geq 1$ year) and (vi) previously being treated unsuccessfully with clomiphene citrate (failure to ovulate or to conceive in six ovulatory cycles). Study approval was obtained from the human subjects committee of the Erasmus Medical Center and informed consent was obtained from all subjects.

Prior to initiation of ovulation induction, standardized initial clinical, sonographic and endocrine screening was performed as described previously (15). Transvaginal sonographic (TVS) screening included assessment of the ovarian stroma echogenicity, ovarian volume $(\mathrm{ml})$ and total number of follicles (both ovaries), as described previously (11). Sonographic monitoring was performed by a single observer (E v S), using an ultrasound machine (model EUB-415; Hitachi Medical Corp., Tokyo, Japan) with a $6.5 \mathrm{MHz}$ transvaginal transducer. Endocrine screening included serum assays for FSH, luteinizing hormone, estradiol (E2), androstenedione, testosterone, sex-hormone binding globulin and insulin-like growth factor-I (IGF-I), as described previously (16). Hormone assays used and the intra- and inter-assay coefficients of variation for these assays have all been described previously (11, 16).

\section{Treatment protocol and monitoring of ovarian response}

Three to five days after a spontaneous or progestageninduced withdrawal bleed, daily i.m. injections of human menopausal gonadotropin (HMG, Humegon; NV Organon Int., Oss, The Netherlands) were commenced. Monitoring of ovarian response was performed every 2 or 3 days until human chorionic gonadotropin (hCG) was administered. A single i.m. dose of $5000 \mathrm{IU}$ hCG (Pregnyl; NV Organon Int.) was administered on the day at least one follicle $\geq 18 \mathrm{~mm}$ was visualized by TVS. If more than three follicles $\geq 16 \mathrm{~mm}$ were present, stimulation was cancelled. Mid-luteal progesterone levels above $15 \mathrm{nmol} / \mathrm{l}$ indicated ovulation.

\section{Step-down protocol}

The starting dose of FSH was fixed on 150 IU per day $(75 \%)$ or modified based on former experience with individual FSH dosage response. The first decrease in dose by half an ampoule/day was carried out when at least one follicle $\geq 10 \mathrm{~mm}$ in diameter was visualized. Further dose decreases of $37.5 \mathrm{IU}$ per day were performed every 3 days to a minimum dose of $75 \mathrm{IU} /$ day if follicular growth continued. This dose was maintained until the day on which hCG was administered. If no ovarian response was observed after 5 days the initial dose was increased by half an ampoule/day. If follicular growth remained absent over the following 10 days (two incremental steps of half an ampoule/day), further medication was withheld and the cycle was cancelled. No luteal support was provided.

\section{Data analysis}

Patients were considered to be clomiphene-resistant anovulatory (CRA) when they failed to ovulate in response to a daily dose of up to $150 \mathrm{mg}$ from cycle day 3 to 7 during at least 3 consecutive cycles. The formula to predict the effective individual FSH starting dose used was: $\left[4 \mathrm{BMI}\left(\mathrm{kg} / \mathrm{m}^{2}\right)\right]+[32$ CRA (yes $=1$ or

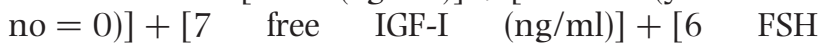
$(\mathrm{IU} / \mathrm{l})]-51$, where BMI = body mass index (14). We used the F-test for ANOVA for comparison of patient groups divided by day of first step-down ( $\leq 3$ days, $>3$ days or constant/step-up) and difference between actual starting dose and predicted starting dose. Kaplan-Meier survival analysis was used to estimate the correlation between the difference in starting dose as compared with the predicted starting dose (too high or too low) and the day of the first step-down.

\section{Results}

Fifty-six patients were treated with exogenous gonadotropins according to a step-down dose regimen. Patient characteristics: median age 29 (range 20-40) years; median BMI 26 (range 19-40) $\mathrm{kg} / \mathrm{m}^{2}$; median duration of infertility 1.6 (range $2-7$ ) years; $24 \%$ amenorrheic, $49 \%$ did not ovulate on clomiphene citrate. Endocrine characteristics (mean and range): FSH 4.7 (1.4-9.0) IU/l; E2 225 (120-378) pmol/l; testosterone $2.5(1.2-4.6) \mathrm{nmol} / \mathrm{l}$ and IGF-I $225(64-384) \mathrm{ng} / \mathrm{ml}$. Treatment outcome is shown in Table 1. Patients were divided into three groups according to the day of the first decrease in gonadotropin dose: (a) early stepdown, treatment day 3 or earlier $(n=33,59 \%)$; (b) standard step-down, day 4 or later $(n=13,23 \%)$; (c) no step-down, gonadotropin starting dose kept constant 
Table 1 Treatment outcome per started cycle in 56 patients treated with exogenous gonadotropins according to a step-down dose regimen in the first induction cycle.

\begin{tabular}{ll}
\hline Induction cycles $(n)$ & 56 \\
Treatment duration (days and range) & $10(6-22)$ \\
Monofollicular growth on hCG day & $65 \%$ \\
Cycles cancelled (no response) & $4 \%$ \\
Cycles cancelled (hyperresponse) & $8 \%$ \\
Ovulation rate & $85 \%$ \\
OHSS & 0 \\
Pregnancies & $19 \%$ \\
Multiple pregnancies & $17 \%$ \\
\hline
\end{tabular}

or increased ( $n=10,18 \%)$. Patients in group (a) had an average starting dose 28.5 IU above the predicted effective starting dose, in group (b) 13 IU above the predicted starting dose and in group (c) 43 IU under the predicted starting dose (Fig. 1). A significant correlation between day of first step-down and the difference between starting dose and individual predicted response-dose was observed $(P<0.0001$, F-test for ANOVA). Kaplan-Meier analysis comparing the day on which the first step-down was performed between the group in which the starting dose was too high with the group in which it was too low, as compared with the predicted starting dose, was also significantly different $(P=0.0072)$.

\section{Discussion}

In the present study, the tested prediction model accurately identified those subjects in whom the starting dose of FSH had been too high, appropriate or too low. Previously, prediction models have been applied to several areas of reproductive medicine. Examples include the prediction of spontaneous pregnancy in subfertile couples $(17,18)$ and the response to clomiphene induction of ovulation (16). A prediction model may be of particular value in the context of ovulation induction with gonadotropins. Since the individual FSH threshold is unknown and varies greatly, this may result in prolonged treatment. When the FSH threshold is finally reached the FSH window has become wide enough to facilitate multiple follicle growth resulting in an increased risk of multiple pregnancies and OHSS. To minimize these life-threatening risks, it may be useful to estimate the individual FSH threshold before starting treatment.

A first step towards individualizing the FSH dose was to start ovulation induction with an initial 'dose-finding' cycle according to a low-dose step-up regimen (19). In this way, for every individual patient, the effective dose resulting in ongoing follicular growth could be determined. This initial ovulation induction cycle was followed by a cycle applying the step-down regimen. This approach resulted in a significant reduction in complications and a similar cumulative pregnancy rate when compared with a step-down dose starting cycle (van Santbrink, unpublished observations). However, the inconvenience and costs of a 'dose-finding' induction cycle were introduced. A second step was the development of a prediction model from this patient group, treated according to a 'dose-finding' low-dose step-up regimen, for the effective FSH starting dose (14). Using this prediction model, a time consuming 'dose-finding' cycle can be avoided.

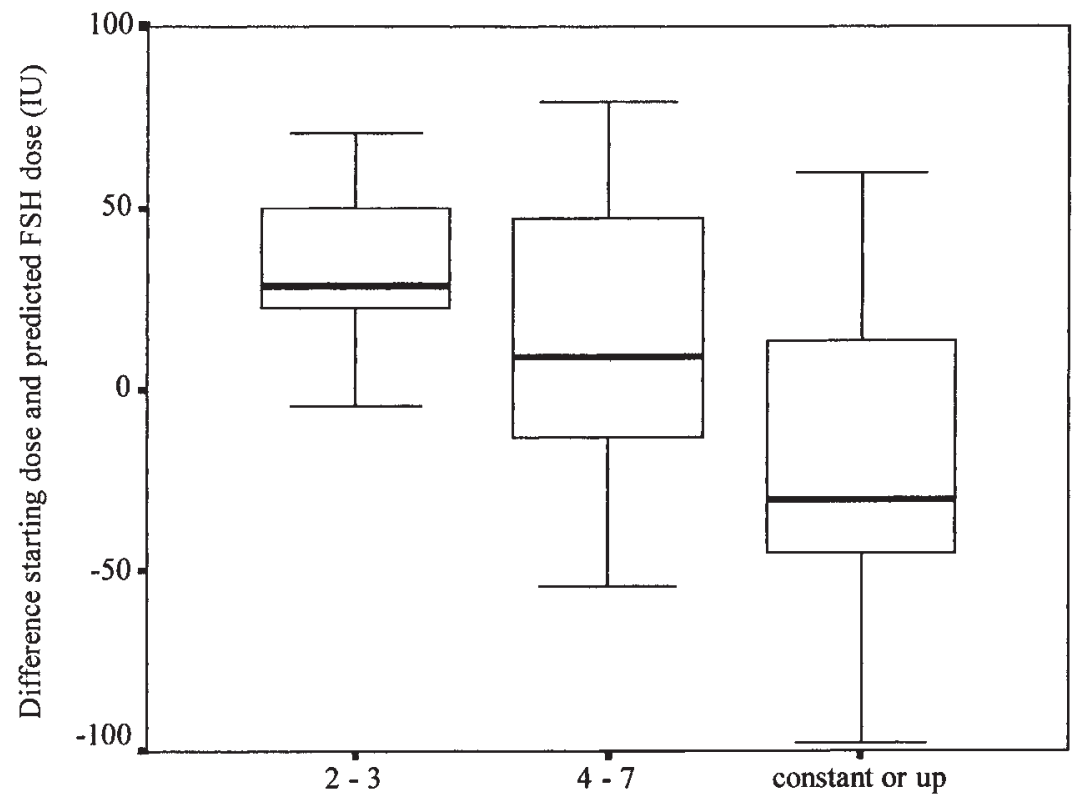

First step-down (day)
Figure 1 Box and whisker plots of normogonadotropic anovulatory patients $(n=56)$ treated with exogenous gonadotropins according to a step-down protocol. Patients are divided into three groups based on the day a first step-down was made: (a) within 3 days (59\%), (b) between 4 and 7 days (23\%) and (c) dose was kept constant or increased $(18 \%)$. For each group the difference between the actual starting dose and the predicted starting dose (according to the prediction model) is shown. Differences between groups were significant $(P<0.001$, F-test for ANOVA). 
In the present study we used this prediction model in a patient group treated according to a step-down regimen in which for most patients $(75 \%)$ the starting dose was fixed on $150 \mathrm{IU} /$ day. A clear correlation between starting dose predicted by the model and the day of first step-down was established. In other words, when the starting dose of FSH was higher than the predicted effective dose, the FSH threshold was reached within 3 days, resulting in an early visualization of a dominant follicle (diameter $>9 \mathrm{~mm}$ ) and a relatively early step-down had to be made. Patients in whom the used starting dose was the predicted effective dose had the first step-down between day 3 and 5 of the induction. The presumption that a static formula can predict the ideal response-dose for an individual patient implicates that within a certain small range (cycle to cycle variation) this dose is constant. However, the tested model is developed from a first low-dose stepup induction cycle so we do not know if this model is also suitable for the following induction cycles. Until this is known, fine-tuning of the starting dose in the following cycles should be done by sonography.

In conclusion, this study provides clinical support for the concept that the effective individual FSH responsedose for normogonadotropic anovulatory women undergoing exogenous gonadotropin ovulation induction with a step-down protocol can be predicted. A prospective trial is now underway to confirm whether such an approach may result in more effective patient treatment protocols, reduced complication risks and health-economic benefits.

\section{Acknowledgements}

This study was financially supported by Stichting Voortplantingsgeneeskunde, Rotterdam.

\section{References}

1 Navot D, Bergh PA \& Laufer N. Ovarian hyperstimulation syndrome in novel reproductive technologies: prevention and treatment. Fertility and Sterility 199258 249-261.

2 Derom C, Derom R, Vlietinck R, Maes H \& van den Berghe H. Iatrogenic multiple pregnancies in East Flanders, Belgium. Fertility and Sterility $199360493-496$.

3 Brown JB. Pituitary control of ovarian function - concepts derived from gonadotrophin therapy. Australian and New Zealand Journal of Obstetrics and Gynaecology 197818 46-54.

4 Fauser BC \& van Heusden AM. Manipulation of human ovarian function: physiological concepts and clinical consequences. Endocrine Reviews 199718 71-106.

5 Taymor ML, Sturgis SH, Goldstein DP \& Lieberman B. Induction of ovulation with human postmenopausal gonadotropin. Effect of varying dosage schedules on estrogen and pregnanediol excretion levels. Fertility and Sterility $1967 \mathbf{1 8} 181-190$.

6 Thompson CR \& Hansen LM. Pergonal (menotropins): a summary of clinical experience in the induction of ovulation and pregnancy. Fertility and Sterility $197021844-853$.

7 Seibel MM, Kamrava MM \& McArdle C. Treatment of polycystic ovary disease with chronic low-dose follicle stimulating hormone: biochemical changes and ultrasound correlation. International Journal of Fertility 198429 39-43.

8 van Santbrink EJ, Donderwinkel PF, van Dessel TJ \& Fauser BCJM. Gonadotrophin induction of ovulation using a step-down dose regimen: single-centre clinical experience in 82 patients. Human Reproduction $1995101048-1053$.

9 Hamilton-Fairley D, Kiddy D, Watson H, Safe M \& Franks S. Lowdose gonadotrophin therapy for induction of ovulation in 100 women with polycystic ovary syndrome. Human Reproduction 19916 1095-1099.

10 Fauser BC, Donderwinkel P \& Schoot DC. The step-down principle in gonadotrophin treatment and the role of GnRH analogues. Baillières Clinical Obstetrics and Gynaecology 19937 309-330.

11 van Santbrink EJ, Hop WC, van Dessel TJ, de Jong FH \& Fauser BCJM. Decremental follicle-stimulating hormone and dominant follicle development during the normal menstrual cycle. Fertility and Sterility $19956437-43$.

12 Schoot DC, Hop WC, Pache TD, de Jong FH \& Fauser BC. Growth of the dominant follicle is similar to normal in patients with gonadotrophin-stimulated PCOS exhibiting monofollicular development during a decremental dose regimen. Acta Endocrinologica $1993129126-129$.

13 van Santbrink EJ \& Fauser BC. Urinary follicle-stimulating hormone for normogonadotropic clomiphene-resistant anovulatory infertility: prospective, randomized comparison between low dose step-up and step-down dose regimens. Journal of Clinical Endocrinology and Metabolism 199782 3597-3602.

14 Imani B, Eijkemans MJ, Faessen GH, Bouchard Ph, Giudice LC \& Fauser BCJM. Prediction of the individual follicle-stimulating hormone threshold for gonadotropin induction of ovulation in normogonadotropic anovulatory infertility: an approach to increase safety and efficiency. Fertility and Sterility 200277 $83-90$.

15 van Santbrink EJ, Hop WC \& Fauser BC. Classification of normogonadotropic infertility: polycystic ovaries diagnosed by ultrasound versus endocrine characteristics of polycystic ovary syndrome. Fertility and Sterility $199767452-458$.

16 Imani B, Eijkemans MJ, te Velde ER, Habbema JD \& Fauser BCJM. Predictors of chances to conceive in ovulatory patients during clomiphene citrate induction of ovulation in normogonadotropic oligoamenorrheic infertility. Journal of Clinical Endocrinology and Metabolism 199984 1617-1622.

17 Snick HK, Snick TS, Evers JL \& Collins JA. The spontaneous pregnancy prognosis in untreated subfertile couples: the Walcheren primary care study. Human Reproduction 199712 1582-1588.

18 Eimers JM, te Velde ER, Gerritse R, Vogelzang ET, Looman CW \& Habbema JD. The prediction of the chance to conceive in subfertile couples. Fertility and Sterility $19946144-52$.

19 van Santbrink EJP. Decremental follicle-stimulating hormone and single dominant follicle selection in the human. PhD Thesis, Erasmus University, Rotterdam, The Netherlands, 1998.

Received 5 April 2002

Accepted 10 May 2002 